Advances in Radio Science (2004) 2: 87-92

(C) Copernicus GmbH 2004

\title{
Susceptibility and hardening of electronic systems to fast transient threats: new challenges ahead
}

\author{
F. Sabath \\ Wehrwissenschaftliches Institut für Schutztechnologien - ABC-Schutz, Munster, Germany
}

\begin{abstract}
The field of susceptibility and hardening of electronic systems to transient threats has experienced a significant growth during the past ten years. Driven by the development in the area of non-lethal electromagnetic weapons it has become necessary to extend the classical set of transient threats, consisting of LEMP, ESD and NEMP, by a fast transient threat with an extreme bandwidth. The investigation of the susceptibility to those UWB threats, characterized by a bandwidth of more than a quarter of the center frequency, rise times of less than 200 ps and pulse durations in the ns regime, is of special interest. This paper presents an overview of current challenges of the hardening against UWB threats. It discusses recent research trends in transient susceptibility measurements, protection concepts and methods of analysis.
\end{abstract}

\section{Introduction}

Electronic components and subsystems (e.g. microprocessor boards) are essential parts of modern civil and military systems like airplanes, communication, traffic management or safety systems. Since electronic components began to control the safety critical functions, the concern grew over the vulnerability of electronic systems. Therefore the susceptibility of critical systems is of vital interest because a setup or failure in these systems could cause major accidents or economic disasters. The increase of non-metallic materials like carbon-fiber composite as well as the decrease of the signal levels result in a decreased susceptibility level of electronic systems.

Wideband and ultra-wideband (UWB) technologies have achieved notable progress in recent years, aided immeasurably by improvements in solid-state manufacturing, computers, and digitizers. As a result, wideband systems that were difficult or impossible to build ten years ago are now being used for an increasingly wide variety of applications including collision avoidance radar, concealed object detection, and communications. UWB systems are also being used for mine clearing, oil pipeline inspections, archeology, and geology as well as electronic effects testing. With the advent of UWB sources capable of producing output powers in the GW range, there has been interest in using UWB devices in military defense applications to disrupt or destroy offensive electronic systems. In numerous publications it has been reported that terrorists have the possibility to interrupt and/or damage sensitive electronics by generating Intentional Electromagnetic Interferences (IEMI) (Wik et al., 1999; Radasky, 2001; Ianoz and Wimpf, 1999). The investigation of the susceptibility of electronic systems as well as their protection and hardening against UWB threats is of great interest as a consequence.

This paper gives an overview of recent challenges in the area of susceptibility and hardening of electronics against the new fast transient (UWB) threat. The organization of this paper is as follows: A brief overview of transient threats is given in Sect. 2. Aspects of time domain susceptibility tests, like the definition of an appropriate test set-up, measurement techniques, the description of transient coupling mechanism and the assessment of effects, will be discussed in Sect. 3 . Section 4 focuses on the hardening and protection of systems against the new UWB threats. Finally, Sect. 5 discusses common problems, limitations and developments of numerical methods.

\section{Characterization of transient threats}

The classical set of transient threats in the EMC area contains:

- lightning electromagnetic pulse (LEMP)

- nuclear electromagnetic pulse (NEMP)

- electrostatic discharge (ESD) and

- burst. 


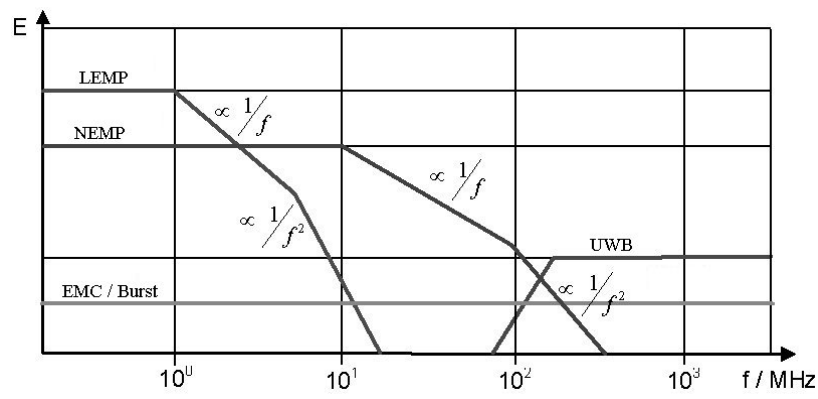

Fig. 1. Spectrum of transient threats.

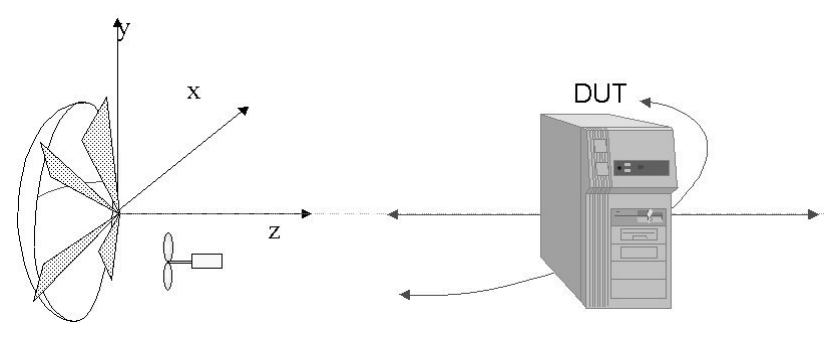

Fig. 2. Principle of the measurement setup.

A characterization of these threats, test and measurement procedures as well as guidelines for the protection have been published in national and international standards and numerous scientific articles. The classical threats are characterized by a double exponential pulse shape with the parameters amplitude, rise time $\left(\tau_{r}\right)$ and the pulse duration (full width half $\max$ time, $\left.T_{F W H M}\right)$. For comparison Table 1 shows in an exemplary way the parameters for typical pulse forms of different transient threats. The process of a standardized characterization is still in progress for UWB pulses (Wik and Radasky, 2002; Wik et al., 1999; Radasky, 2001). Based on results of various experiments and susceptibility investigations an appropriate description is given by a signal with a double exponential shape, a rise time of less than $200 \mathrm{ps,} \mathrm{a}$ pulse width of several ns (2-10 ns) and an amplitude up to $100 \mathrm{kV} / \mathrm{m}$. The established transient EMC environments are compared qualitatively with the emerging UWB waveform in Fig. 1. Due to the fast rise time as well as the short pulse duration the main spectrum starts at $100 \mathrm{MHz}$, a frequency at which the spectrum of classical transient threats like LEPM and NEMP are of vanishing value. This means that the upcoming UWB waveform will threaten electronic systems in a part of the electromagnetic spectrum in which established transient threats could be ignored. Therefore it is necessary to investigate the behavior of classical hardening concepts and protection measures to UWB threats.
Table 1. Parameter of transient threats.

\begin{tabular}{cccc}
\hline Threat & Magnitude & $\tau_{r}$ & $\left.T_{F W H M}\right)$ \\
\hline LEMP & $100 \mathrm{kA}$ & $10 \mu \mathrm{s}$ & $350 \mu \mathrm{s}$ \\
NEMP & $50 \mathrm{kV} / \mathrm{m}$ & $3 \mathrm{~ns}$ & $24 \mathrm{~ns}$ \\
Burst & $2 \mathrm{kV}$ & $5 \mathrm{~ns}$ & $50 \mathrm{~ns}$ \\
ESD & $10 \mathrm{~A}$ & $700 \mathrm{ps}$ & $100 \mathrm{~ns}$ \\
UWB & $100 \mathrm{kV} / \mathrm{m}$ & $<200 \mathrm{ps}$ & $<10 \mathrm{~ns}$ \\
\hline
\end{tabular}

\section{Time domain susceptibility tests}

\subsection{Test setup}

Investigations of the effects of fast transient electromagnetic fields on modern electronic systems generally use a measurement setup consisting of a high power pulse source, a pulse forming network and a field generating device. Field pulses with a $10-90 \%$ rise time in the 100 ps regime and a $50 \%$ pulse width of less than $10 \mathrm{~ns}$ can be generated by - TEM-waveguides (open strip line, GTEM-Cell,...) - impulse radiating antennas (IRAs)

- Small conical antennas.

On the one hand the TEM-waveguides are characterized by a non-dispersive transfer function and a field homogeneity of high quality. On the other hand the behavior of higher order modes limits the useable size of the test volume. In contrast to the TEM-waveguides the antennas are able to illuminate whole systems, but they are characterized by a derivative transfer function. In general time domain susceptibility measurements are similar to the common measurement procedures in frequency domain. Both procedures start with a calibration of the incident test field in absence of the device under test (DUT). Following this the DUT is located in the center of the usable test volume and illuminated by the test field. The amplitude of the test field is increased gradually until malfunctions or destructions can be observed. Due to uncertainties of the pulse sources the amplitude of the test field should be monitored by a reference probe during the whole test.

As the amplitude range of the output voltage of the available high power pulse sources is restricted or the amplitude variation causes a change of the pulse shape, the field amplitude can only be scaled by the use of attenuator or changes of the distance between the antenna and the DUT. In the case attenuators are used, they must be able to diminish nondispersievly voltage pulses with a pulse amplitude of several $100 \mathrm{kV}$ and a bandwidth up to $10 \mathrm{GHz}$. In the standard measurement procedure the amplitude of the test field pulse is varied by changing the distance between the antenna and the DUT (Fig. 2).

For impulse radiating antennas as well as antenna array with an aperture diameter of less than $2 \mathrm{~m}$ far field conditions are assumed for distances larger than $8 \mathrm{~m}$ with regard to $\mathrm{cw}$ measurement procedures. This assumption neglects the 

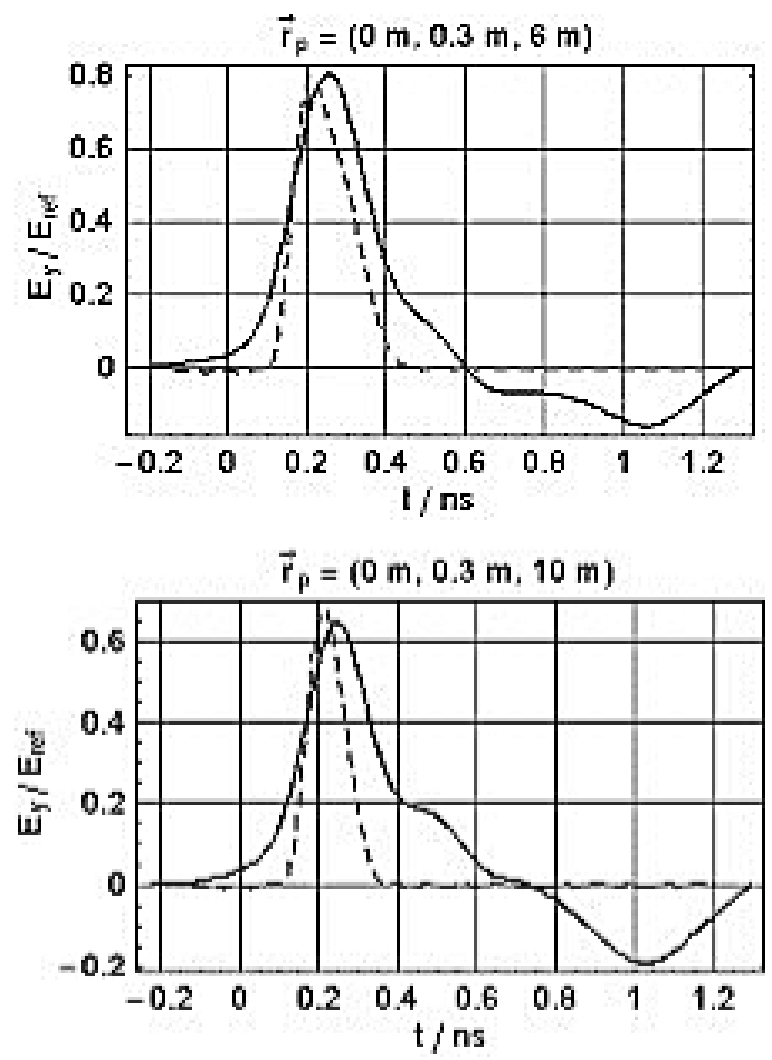

Fig. 3. Field pulse at various distances (solid: measured; dashed: computed).

influence of transient near field effects on the shape and the spectrum of the field pulse. I have shown (Sabath and Garbe, 2002) that the dimension of antenna systems and the wave propagation lead to a special kind of dispersion. As an example Fig. 3 shows UWB field signals at various distances from the IRA. In Sabath and Garbe (2002) I have explained that this dispersion has the potential to effect susceptibility tests seriously. For susceptibility investigations it is necessary to assess this distortion of the radiated signal with regard to the spectrum of the feeding signal and the spectral susceptibility behavior of the DUT.

\subsection{Measurement techniques}

An other challenge is given by the measurement of UWB waveforms, particularly in case of UWB fields radiated from an IRA. The extreme bandwidth of the UWB waveform requires a measurement chain with a linear transfer function over a bandwidth from the $300 \mathrm{MHz}$ up to $10 \mathrm{GHz}$. Following the challenges are explained by taking a closer look on a measurement chain consisting of an impulse source ${ }^{1}$ connected to an IRA and a field sensor ${ }^{2}$.

For the connection between the impulse source and the antenna (IRA) cable are available that are able to transmit the

\footnotetext{
${ }^{1}$ e.g. PBG 3: $U=12,5 \mathrm{kV}, \tau_{r}=100 \mathrm{ps}, T_{F W H M}=2,5 \mathrm{~ns}$

2e.g. AD-10: $A_{e q}=10^{-4} m^{2}, f_{3 d B}>10 \mathrm{GHz}$
}
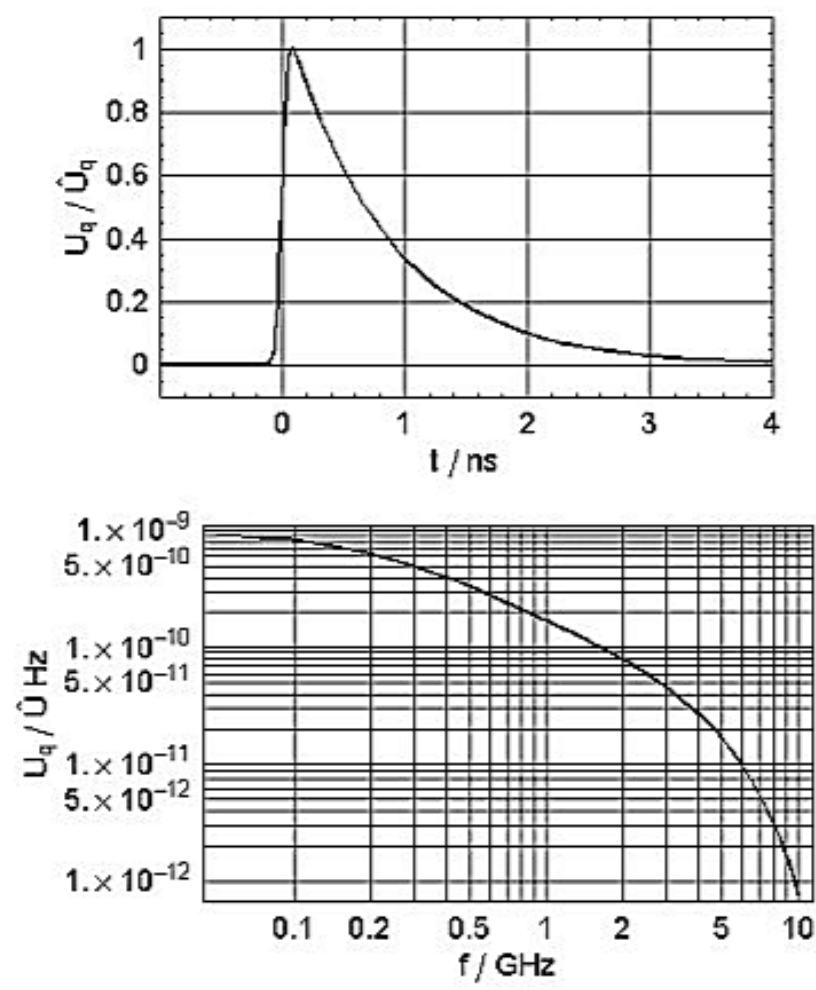

Fig. 4. Voltage (above) and spectrum (below) of an impulse generator.

Table 2. Parameter of signals.

\begin{tabular}{lcccc}
\hline Location & $\tau_{r}$ & $f_{\text {low }}$ & $f_{\text {high }}$ & $B$ \\
\hline Source & $100 \mathrm{ps}$ & $0,00 \mathrm{GHz}$ & $0,30 \mathrm{GHz}$ & $0.30 \mathrm{GHz}$ \\
Field & $65 \mathrm{ps}$ & $0,10 \mathrm{GHz}$ & $4,95 \mathrm{GHz}$ & $4,85 \mathrm{GHz}$ \\
Sensor & $54 \mathrm{ps}$ & $1.28 \mathrm{GHz}$ & $7.66 \mathrm{GHz}$ & $6,38 \mathrm{GHz}$ \\
\hline
\end{tabular}

waveform (Fig. 4), particularly the short rise time, and withstand the high voltage amplitude. For the monitoring of the waveform one could use two kinds of classical probes. The first kind of probes are able to measure high voltage signals but only a rise time in the ns regime. The sensors of the second kind are designed for short rise times but they are not able to withstand the high voltage. In Weber and ter Haseborg (2004) Weber presented a novel set-up consisting of an oil filled coaxial line combined with a conical field sensor. Using this voltage probe, called picoTEM cell, he was able to monitor UWB signals with an amplitude up to $12,5 \mathrm{kV}$.

The derivative behavior of the IRA results in a shift of the spectrum to higher frequencies as well as to a wider bandwidth (Fig. 5). The comparison of the characteristic frequencies (Table 2) clarifies that the field has a bandwidth that is one dimension larger than that of the feeding voltage pulse. In the example shown the IRA will radiate a field pulse with a bandwidth of $4,85 \mathrm{GHz}$. The next challenge in the area of measuring UWB signals is to measure the rise time as well as 

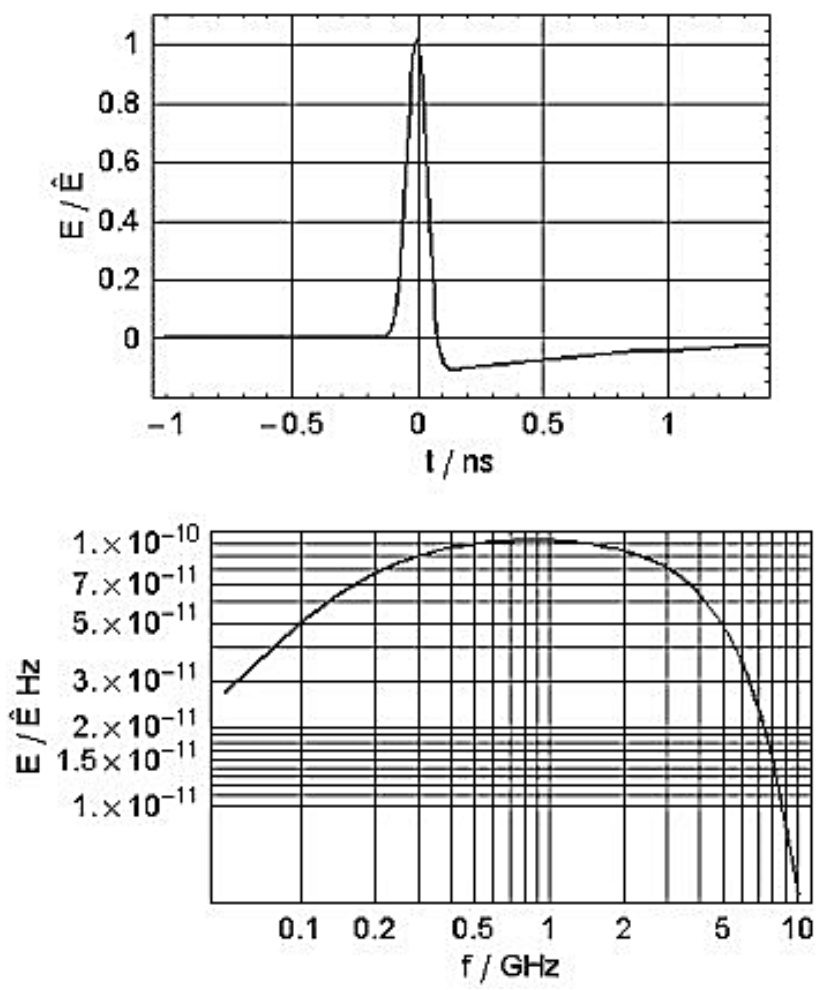

Fig. 5. Signal (above) and spectrum (below) of the electric field strength generated by an IRA.

the energy of this field signal correctly. Due to the resonant behavior of large field sensors (like dipoles) the field strength will be measured using electrical small conical sensors or small TEM based sensors. Due to the constant transmission TEM based sensors are able to measure the energy correctly without any mathematical post processing. The lower bandwidth limits the application of the TEM based sensors. In contrast to this, the conical sensors are characterized by a larger bandwidth. But the derivative behavior in combination to a signal noise leads to an information loss concerning the energy. In the example the output voltage of the used AD10 sensor (Fig. 6) is characterized by a bandwidth of 6,38 $\mathrm{GHz}$. This bandwidth is larger than that of any commercially available fiber optic link system or cable system. The actual parameters of field signal could only be computed by a mathematical correction of the non-perfect transmission of the connection used. A widely used method is the application of Fourier transformation, correction in the frequency domain and computation of the corrected time function via inverse Fourier transformation. In case of UWB signals the correct calculation of the short rise time requires frequencies in the $10 \mathrm{GHz}$ regime. The reconstruction of the energy content needs frequencies in the $\mathrm{MHz}$ regime. Particularly the needed length of the measurement window in combination with the extreme short pulse challenges the measurement method. The impact of noise, reflections caused by the environment and post pulse radiation of the antenna increase the problem. Wavelet transformation and forward modeling
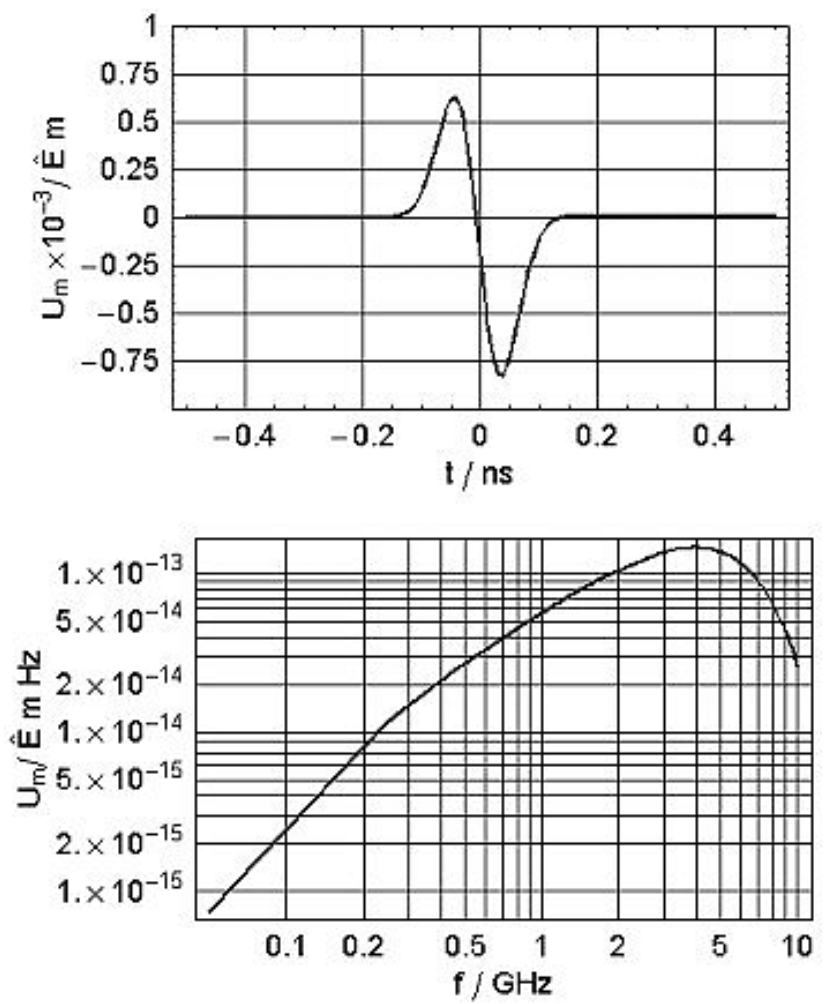

Fig. 6. Signal (above) and spectrum (below) of the output voltage of a D-dot sensor (AD-10).

techniques are expected to provide major contributions for the solution of this technical problem.

\subsection{Modelling and assessment}

Beside the testing and measuring the assessment of effects and the modelling of the transient coupling are importand elements of a systematic hardening procedure. The assesment includes the two aspects: functional assessment and identification of critical parameters. With the functional assessment the impact of an observed malfunction or destruction is analyzed from the operational point of view. Typical results are safety critical interference, functional critical interference, limiting interference or uncritical interference. The more challenging part of the assesment is the identification of critical parameters. This means answering the question: Which parameter of the incident waveform caused the observed effect? Contrary to narrow-band signals ${ }^{3}$ a transient waveform is characterized by a set of the seven parameters:

- amplitude

- rise time $\tau_{r}$

- pulse width $T_{F W H M}$

- pulse repetition rate

- energy

- distribution of the spectral energy density.

\footnotetext{
${ }^{3}$ which are characterized by only three indipendent pararameter amplitude, frequency and quality (Q)
} 


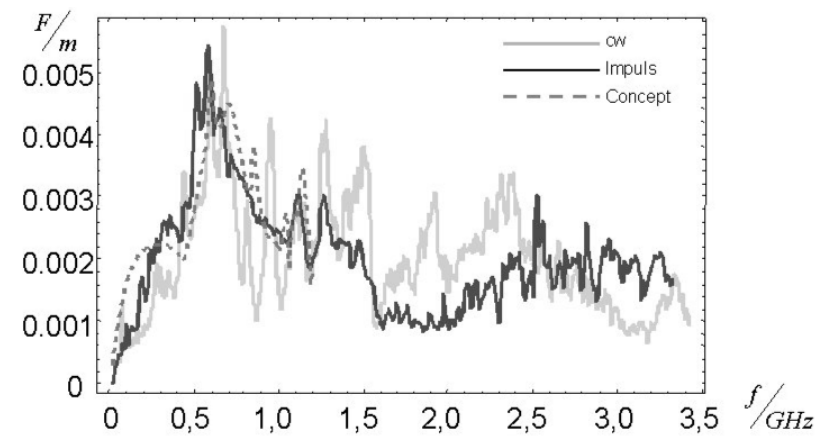

Fig. 7. Amplitude of a transfer function of a generic system.

It has to be noticed that these parameters are not independent. Particularly for most of the malfunctions and the degradation of service are various candidates are under discussion. Current status of investigation and new results on this topic are presented in Camp and Garbe (2003); Esser and Smailus (2003).

In order to modell the coupling of an incident field into electronic systems the identification of coupling path and their description via tranfer factor ${ }^{4}$ or transfer function (Fig. 7) is a commonly used methodology. In case of small simplifyed or generic systems characterized by linear behavior and a limited number of possible copling paths the transfer function could be determined by measurements or numerical analysis. The extreme bandwith of UWB waveforms limits the application of this method. If the complexity of real systems and the non-linear behavior of systems of interest are taken into account, the application of exact determined transfer functions are limited on specific locations (e.g. connectors) and academic environment conditions. Currently norm based and statistical models are discussed as key elements for the prediction of the system response of an UWB interaction (Nitsch et al., 2003).

\section{Hardening and protection}

In this section I intend to give a short overview about challenges in the area of hardening and protection against UWB threats. A detailed presentation of the recent state of knowledge could be found in Krzikalla and ter Haseborg (2003); Weber and ter Haseborg (2002b); Krzikalla et al. (2003); Weber et al. (2004).

In order to protect electronic systems against unwanted and intentional electromagnetic interferences with high energy and amplitudes non-linear protection elements like spark gaps, gas discharge tubes, varistors or Z-diodes are used commonly. Most of these protection elements are designed for the classical transient threats (LEMP, NEMP, ESD and burst). The combination of significant amplitudes with rise times in the Pico second range are a nontrivial challenge for all kinds of protection measures. Protection el-

\footnotetext{
4 in case of narrow band waveforms
}
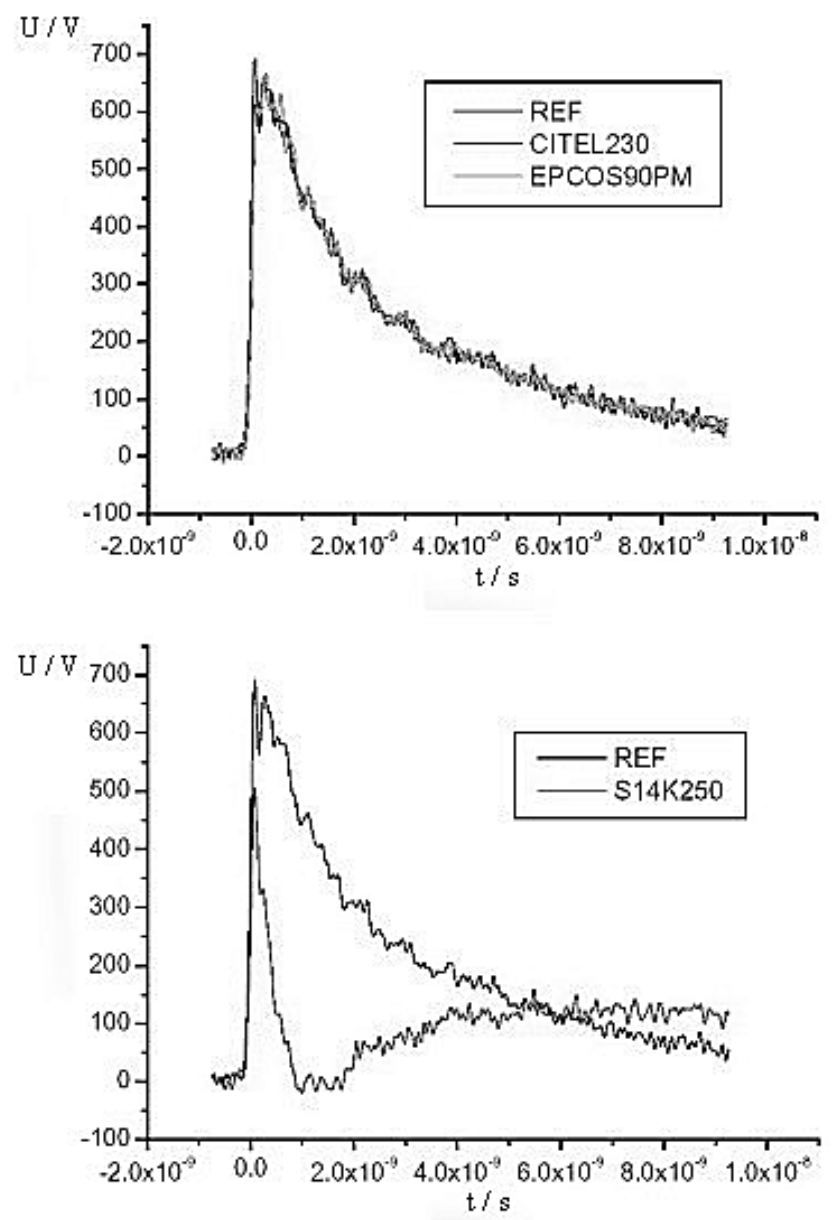

Fig. 8. Transient response of a gas discharge tubes (above) and a varistor (below, Epcos S14K250).

ements which are applicable for power line protection like spark gaps, discharge tubes and varistors are able to reduce the energy content of an interfering signal. As the respond time of these protection elements is long compared to the rise time of UWB pulses there is no significant amplitude reduction and only a pulse shortening (Fig. 8). In some experiments this behavior results in a dangerous sharpening of the interfering signal. It is remarkable that the response of varistors to UWB signals is dominated by linear effects caused by the capacitance and feed line inductivities.

In contrast to this behavior protection elements for high speed data lines (e.g. Z-diodes, suppression diods) are characterized by short response time, but they can stand high voltage pulses only for a limited time. Weber has investigated the time response of various HF diodes to UWB threats (Krzikalla and ter Haseborg, 2003; Weber et al., 2004). As a result he observed a significant reduction of the transmitted pulse energy.

Due to their high energy handling capability bandpasfilters are expected to provide contributions on the hardening of electronic systems. Investigation of a combination of special linear filters and nonlinear elements could result in an optimized protection concept. 


\section{Numerical computation}

The last aspect of an overall susceptibility concept for electronic systems is the numerical computation of electromagnetic coupling, interfering signals and the response of the threatened system. The analysis of the electromagnetic behavior can be performed exactly by solving Maxwell's equations in time or frequency domain. In particular, for open area problems and linear systems these equations are transformed into integral equations, which reduce the solution to a boundary problem. The frequency dependent coupling behavior of systems under consideration are taken into account by these frequency domain methods (e.g. Method of Moment, MoM). The extreme bandwidth of UWB threats results in long computation times for frequency domain (FD) methods. A disadvantage of FD methods is that they are not able to handle non-linearitys. In contrast to FD methods time domain (TD) methods are designed to consider non-linear behavior. In particular the short pulse duration in combination with a medium response cycle of the system lead to short computation time. The frequency dependent coupling behavior and a long respond cycle of the system under consideration describes the worst case for TD methods.

Methods like the PEEC that could be used in both time and frequency domain are expected to provide major contributions for numerical analysis of UWB interaction. In Wollenberg and Kochtetov (2003) Wollenberg shows that a full wave PEEC solver which is integrated in SPICE is successfully applicable to problems of UWB intermodulation modeling.

Acknowledgements. The author thank his colleagues Dr. U. Schenk and D. Nitsch for continuing discussions and helpful comments.

\section{References}

Abrams, M.: The dawn of the E-Bomb, IEEE Spectrum, 40, 2003.

Camp, M. and Garbe, H.: Susceptibility of Personal Computer Systems to Electromagnetic Pulses with Double Exponential Character, Advances in Radio Science, Copernicus GmbH, 2, 2003.

Esser, N. and Smailus, B.: Measuring the Upset of CMOS and TTL due to HPM-Signals, Advances in Radio Science, Copernicus $\mathrm{GmbH}, 2,2003$.

Ianoz, M. and Wimpf, H.: Modelling and simulation methods to assess EM terrorism effects, in Proceedings of the 13th International Zurich Symposium on EMC, Zürich (CH), 13, 1999.
Krzikalla, R. and ter Haseborg, J.-L.: HPM protection on HF transmission lines, Advances in Radio Science, Copernicus GmbH, 2, 2003.

Krzikalla, R., Weber, T., and ter Haseborg, J.-L.: Interdigital microstrip filters as protection devices against ultrawideband pulses, in Proceedings of the International IEEE Symposium on EMC, Istanbul (Turkey), 2003.

Nitsch, D., Sabath, F., Schmidt, H.-U., and Braun, C.: Comparison of the High-Power Microwave and Ultra Wide Band Susceptibility of modern Microprocessor Boards, in Proceedings of the 15th International Zürich Symposium on EMC, vol. 15, Zürich $(\mathrm{CH})$, 2003.

Prather, W. D., Baum, C. E., Torres, R. J., Sabath, F., and Nitsch, D. Survey of Worldwide Wideband Capabilities, IEEE Transaction on EMC, in print, 2004.

Radasky, W. E. A.: Intentional Electromagnetic Interference (EMI) - Test data and implications, in Proceedings of the 14th International Zurich Symposium on EMC, Zürich (CH), 14, 2001.

Sabath, F. and Garbe, H.: Impact of near field dispersion on time domain susceptibility tests, Advances in Radio Science, Copernicus $\mathrm{GmbH}, 1,2002$.

Sabath, F., Bäckström, M., Nordström, B., Serafin, D., Kaiser, A., Kerr, B., and Nitsch, D.: Overview of High Power Microwave Narrow Band Test Facilities, in Procceedings of the ICEAA 03, Turino, Italy, 2003.

Weber, T. and ter Haseborg, J.-L.: High-Voltage short-pulse measurements, in Proceedings of the AMEREM 2002, Annapolis (MA), 2002a.

Weber, T. and ter Haseborg, J.-L.: Hardening of electronics against transmission line coupled UWB-Signals, in Proceedings of the XXVIIth URSI General Assembly, Maastricht (NL), 27, $2002 \mathrm{~b}$.

Weber, T. and ter Haseborg, J.-L.: Measurement techniques for conducted HPEM signals, IEEE Transaction on EMC, paper in Print, 2004.

Weber, T., Krzikalla, R., and ter Haseborg, J.-L.: Linear and nonlinear filters suppressing UWB pulses, IEEE Transaction on EMC, in print, 2004

Wik, M. W. and Radasky, W. A.: Intentional Electromagnetic Interference (IEMI) - Background and status of the standardisation work in the International Electrotechnical Commission, in Proceedings of the XXVIIth URSI General Assembly, Maastricht (NL), 27, 2002.

Wik, M. W., Gardner, R. L., and Radasky, W. A.: Electromagnetic terrorism and adverse effects of high power electromagnetic environments, in Supplement to proceedings of the 13th International Zürich Symposium on EMC, Zürich (CH), 13, 1999.

Wollenberg, G. and Kochtetov, S. V.: Coupling electromagnetic pulse-shaped waves into wire-like interconnection structures with a non-linear protection - Time domain calculations by the PEEC method, Advances in Radio Science, Copernicus GmbH 2,2003 